\title{
Considerable osteogenic effect around dental implants based on guided bone regeneration technique in plateau low-oxygen regions
}

\begin{abstract}
Purposes: To describe osteogenic effect of two patients with resorption of the alveolar ridgetreated via guided bone regeneration (GBR) technique in plateau low-oxygen regions up to 3000 meters.
\end{abstract}

Methods: We restored the bones defect by placing the bone scraps and bone substitutes (Bio-Oss $\left.{ }^{\circledR}\right)$ on the bone surface surgically. And then, we covered the resorbable collagen membrane above. Moreover, we applied the Double-layer membrane technique to ensure the longer barrier function.

Results: No symptoms of infection around the implants were found in both cases. The two patients achieved good ossification and implanted dentures.

Conclusion: We achieved considerable osteogenic effect around dental implants via GBR technique in the plateau low-oxygen regions up to 3000 meters.

Keywords: plateau, low-oxygen, guided bone regeneration, ossification, dental implant
Volume 8 Issue 6 - 2018

\author{
Bo Qiao,' Fengze Wang,' Rui Zhao,' Tingting \\ Jia,' Fanhao Meng,' Liang Zhu,' Jixian Xin, ${ }^{3}$ \\ Haizhong Zhang \\ 'Oral and Maxillofacial Surgery Department, The Chinese PLA \\ General Hospital, China \\ ${ }^{2}$ Department of stomatology, The 316th Hospital of Chinese \\ People's Liberation Army, China \\ ${ }^{3}$ Department of stomatology, The 22th Hospital of Chinese \\ People's Liberation Army, China
}

Correspondence: Haizhong Zhang, Oral and Maxillofacial Surgery Department, The Chinese PLA General Hospital, No. 28 Fuxing Road, Haidian District, Beijing, China, Tel 010-66938316, Fax 010-66938316 Email doctar30I@aliyun.com

Received: November 14, 2018 | Published: November 23 2018

\section{Introduction}

The number and quality of bone grafts around the implants are the main factors for the stability of the implants. ${ }^{1}$ Severe vertical alveolar bone loss often occurs due to severe chronic periodontitis resulting to tooth extraction. In some cases, the height of the missing teeth bone height is lower than the adjacent alveolar bone. Furthermore, vertical bone augmentation is necessary to recover normal ridge height. ${ }^{2,3}$ Currently, GBR has been used to promote bone to grow rapidly in the gap between the implants and bone. Few documents were reported regarding to GBR technique in the plateau areas due to environmental, economic and medical situation. However, with the development of plateau medicine, the influence of environmental factors, especially hypoxic environment on bone metabolism, have been paid more and more attention. This preliminary observation targeting osteogenic effect of GBR in plateau low-oxygen regions is bound to lay the foundation for further research.
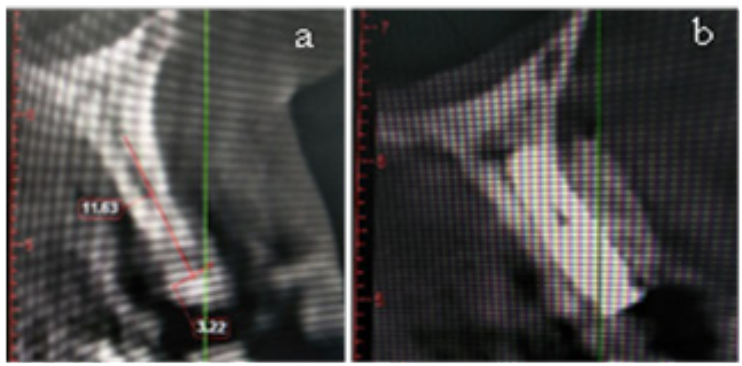

\section{Case report}

\section{Case}

A 48-year-old male patient settled in the plateau area, with a chief complaint of implant denture restoration. The maxillary right lateral incisor of the patient was removed due to periodontitis. (Health status: smoking for 21 years, fasting blood glucose: $7.2 \mathrm{mmol} / \mathrm{L}$ ). Surgical approach: we made a midline incision on the edentulous alveolar crest, adding a vertical loose incision. Moreover, the full thick mucoperiosteum flap was developed. We placed an Osstem implant $\left(3.3^{*} 10 \mathrm{~mm}\right)$ after flap opened. The buccal bone defect was surgically treated by placing bone scraps and bone substitutes $\left(\mathrm{Bio}^{-O} \mathrm{ss}^{\circledR}\right)$ and covered with a resorbable collagen membrane $\left(\right.$ Bio-Gide $\left.{ }^{\circledR}\right)$. Doublelayer membrane technique was used to ensure longer barrier function (Bosshard and Schenk, 2010). CBCT showed preoperative and postoperative results (Figure 1a) (Figure 1b). Clinical photographs of preoperative and of 6 months after the operation (Figure1c,) (Figure 1d).

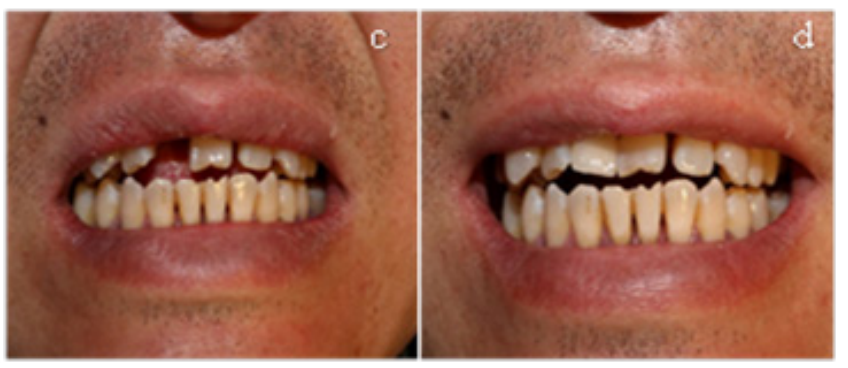

Figure I (a) CBCT of preoperation. (b) CBCT of postoperation. (c) Clinical photograph of preoperation. (d) Clinical photograph of 6 months after the operation. 


\section{Case2}

General population: A 56-year-old male patient settled in the plateau area complained pain of his mandibular first molar (Health status: none smoking, fasting blood glucose: $7.8 \mathrm{mmol} / \mathrm{L}$ ). The two mandibular first molars were removed due to vertical root fracture and residual root (Figure 2a). Four months later, the alveolar fossa of 36-tooth was well healed. However, the 46-tooth's alveolar fossa was healed poorly (Figure $2 b$ ).

Surgical plan: The site of left mandibular first molar was implanted with Megagen implant $(4.5 * 10 \mathrm{~mm})$. Moreover, the site of right mandibular first molar was placed bone scraps and bone substitutes $\left(\right.$ Bio-Oss $\left.{ }^{\circledR}\right)$ and been covered with a resorbable collagen membrane (Bio-Gide ${ }^{\circledR}$ ) above. Double-layer membrane technique was applied. Panoramic radiograph showed expected result after operation (Figure 2c). After six months, the result showed that the alveolar fossa of right mandibular first molar had a good osteogenic effect (Figure 2d). The left mandibular first molar's abutment was repaired with an all-ceramic crown and the site of right mandibular first molar was implanted with Megagen implant $\left(4.5^{*} 08 \mathrm{~mm}\right.$ ) (Figure 2e). After three months, the right mandibular first molar's abutment was repaired with an all-ceramic crown. The patient was reviewed every three months in the first year, and every six months thereafter (Figure $2 \mathrm{f}$ ).
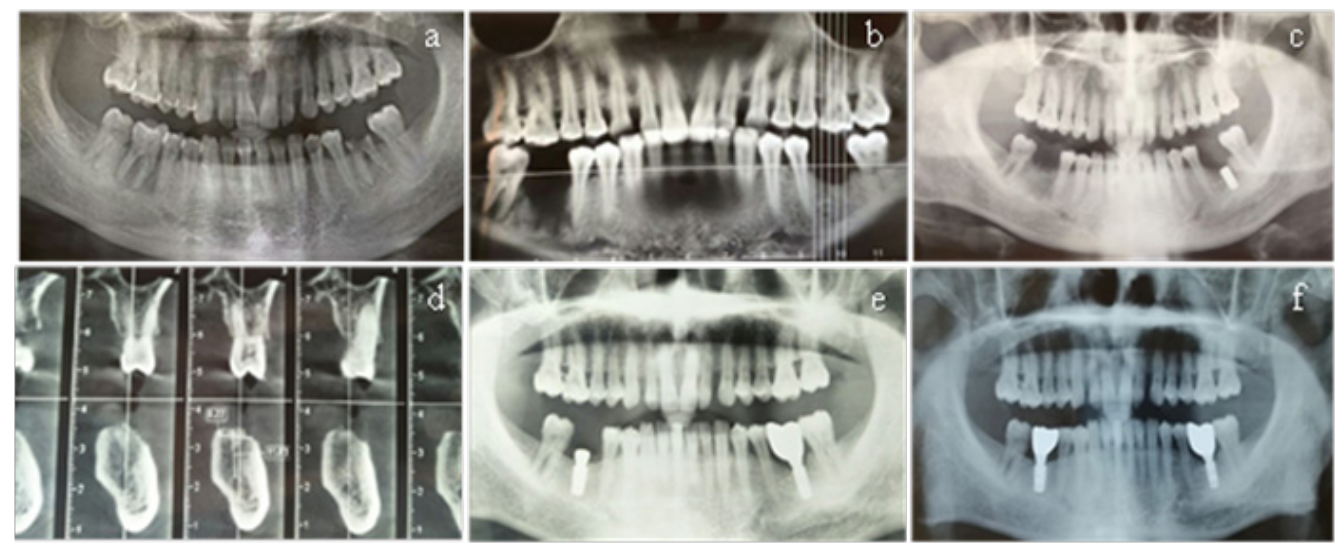

Figure 2(a) Panoramic radiograph before extraction. (b) Four months later after extraction. (c) Panoramic radiograph after GBR. (d) Six months later after GBR. (e) Panoramic radiograph showed a considerable osteogenic effect around dental implant. (f) Stableosteogenic effect after two years.

\section{Discussion}

There are several relevant factors influencing the success of dental implants. Among these factors, bone quality and quantity around the implant site play a key role. GBR has been used to promote bone to grow rapidly in the gap between the implants and bone. ${ }^{4}$ In the hypoxic environment, the blood supply around the bone graft area is reduced. The tissue hypoxia is aggravated and the osteoblast differentiation is insufficient. Compared with the plain area, the bone mass of the elderly patients in the highland area is seriously lost, resulting in an increased risk of osteoporosis. However, plateau regions have a drier climate and the incidence of infection is lower, which is a favorable factor for bone grafting. These factors increased the uncertainty of osteogenic effect. Notably, the study regarding to dental implantbone osseointegration in high altitude hypoxic environment up to 3000 meters has not been reported before. Impressively, no symptoms of infection around the implant were found in both cases and all achieved good ossification and implanted dentures. In the future, longterm follow-up and a large-scale randomized clinical investigation will be performed to evaluate the feasibility of GBR technique in plateau low-oxygen regions.

\section{Conclusion}

We achieved considerable osteogenic effect around dental implants based on GBR technique in plateau low-oxygen regions up to 3000 meters.

\section{Acknowledgments}

The authors wish to thank the $22^{\text {th }}$ Hospital of the Chinese People's Liberation Army for great support during patient treatment.

\section{Conflicts of interest}

The author declares that there is no conflicts of interest.

\section{References}

1. Johnson, Trenton B. Effect of guided bone regeneration on bone quality surrounding dental Implants. J Biomech. 2018; 80:166-170.

2. Buser D, Dula K, Belser UC, et al. Localized ridge augmentation using guided bone regeneration. II. surgical procedure in the mandible. Int $J$ Periodontics Restorative Dent. 1995;15(1):10-29.

3. Handelsman M. Surgical guidelines for dental implant placement. $\mathrm{Br}$ Dent J. 2006; 201(3):139-152.

4. Liu J, Kerns DG. Mechanisms of guided bone regeneration: a review. Open Dent J. 2014;8:56-65. 